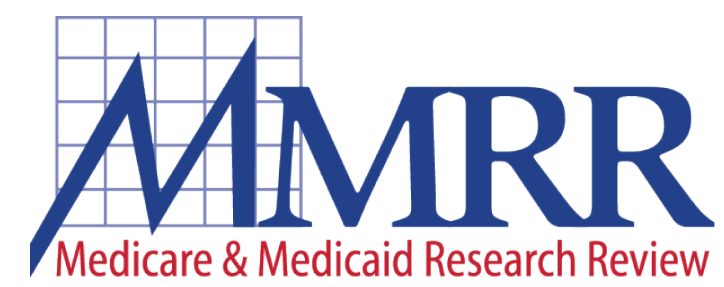

2013: Volume 3, Number 4

A publication of the Centers for Medicare \& Medicaid Services, Office of Information Products \& Data Analytics

\title{
Asthma Medication Ratio Predicts Emergency Department Visits and Hospitalizations in Children with Asthma
}

\author{
Annie Lintzenich Andrews, ${ }^{1}$ Annie N. Simpson, ${ }^{2}$ William T. Basco Jr., ${ }^{1}$ Ronald J. Teufel II, ${ }^{1}$ \\ Medical University of South Carolina \\ ${ }^{1}$ Department of Pediatrics \\ ${ }^{2}$ Department of Healthcare Leadership and Management
}

Objective: To determine if the asthma medication ratio predicts subsequent emergency department (ED) visits and hospital admissions in children.

Design: Retrospective cohort with two year pairs. Setting/Participants: 2007-2009 South Carolina Medicaid recipients with persistent asthma age 2-18. Main Exposure: Controller-to-total asthma medication ratios were calculated for each patient in 2007 and 2008. Ratios range from 0-1 (1 = ideal, $0=$ no controller).

Outcome Measures: 2008 and 2009 asthma related ED visits, hospitalizations, and a combined outcome of ED visit or hospitalization in the subsequent 3, 6 , and 12 month time periods.

Results: 19,512 patients were included. Mean age 8.9 years, $58 \%$ male, and $55 \%$ black. The ratio significantly predicted ED visits and hospitalizations over subsequent 3,6 , and 12 month time periods. The cut-point that maximized the ability to predict visits ranged from $0.4-0.6$. A cutpoint of 0.5 was used in the final models. After controlling for age, race, gender, and rurality, patients with a ratio $<0.5$ were significantly more likely to have a subsequent emergent healthcare visit (OR 1.5-2.0). The ratio retained its predictive ability in both year-pairs for all three outcome variables, in all three time periods, with the exception of the 2008 ratio not predicting 2009 3-month and 6-month hospitalizations.

Conclusions: The asthma medication ratio is a significant predictor of $\mathrm{ED}$ visits and hospitalizations in children. Using a cutoff of $<0.5$ to signal at-risk patients may be an effective way for populations who would benefit from increased use of controller medications to reduce future emergent asthma visits.

Keywords: pediatrics, Medicaid, chronic disease

ISSN: 2159-0354

doi: http://dx.doi.org/10.5600/mmrr.003.04.a05

CPT only copyright XXXX-2012 American Medical Association. All rights reserved.

CPT is a registered trademark of the American Medical Association.

Applicable FARS/DFARS Apply to Government Use.

Fee schedules, relative value units, conversion factors and/or related components are not assigned by the AMA, 


\section{Medicare \& Medicaid Research Review} 2013: Volume 3, Number 4

\section{Mission Statement}

Medicare \& Medicaid Research Review is a peerreviewed, online journal reporting data and research that informs current and future directions of the Medicare, Medicaid, and Children's Health Insurance programs. The journal seeks to examine and evaluate health care coverage, quality and access to care for beneficiaries, and payment for health services.

\section{http://www.cms.gov/MMRR/}

\section{U.S. Department of Health \& Human Services Kathleen Sebelius Secretary}

\section{Centers for Medicare \& Medicaid Services Marilyn Tavenner Administrator}

Editor-in-Chief

David M. Bott, Ph.D.

The complete list of Editorial Staff and Editorial Board members may be found on the MMRR Web site (click link): $\underline{\text { MMRR Editorial Staff Page }}$

Contact: $\underline{\text { mmrr-editors@cms.hhs.gov }}$

Published by the Centers for Medicare \& Medicaid Services.

All material in the Medicare \& Medicaid Research Review is in the public domain and may be duplicated without permission. Citation to source is requested. are not part of CPT, and the AMA is not recommending their use. The AMA does not directly or indirectly practice medicine or dispense medical services. The AMA assumes no liability for data contained or not contained herein.

See attached CMS CPT 2013 end user license

\section{Background}

Asthma is the most common chronic condition affecting children, resulting in frequent emergency department (ED) visits and hospital admissions. (Akinbami, 2006; Akinbami, Moorman, Garbe, \& Sondik, 2009) Despite disease management guidelines and advances in medical treatments, the incidence of emergent health care visits for asthma has remained high. (Akinbami et al., 2012) Even though there is strong evidence that controller medications reduce the frequency of emergent health care visits for asthma, they continue to be underutilized. (Adams et al., 2001; Andrews, Teufel, \& Basco, 2012; Cloutier, Hall, Wakefield, \& Bailit, 2005; Finkelstein, Lozano, Farber, Miroshnik, \& Lieu, 2002; Lieu et al., 1997; Warman, Silver, \& Stein, 2001).

Previous studies have shown that the controller-to-total asthma medication ratio is associated with same year emergent health care utilization and therefore could be considered a reasonable care process measure and/or current disease control measure (Fuhlbrigge et al., 2004; Vernacchio, Trudell, \& Muto, 2013). Schatz et al. (2006) showed that the ratio could also serve as a useful predictor of emergent health care visits for asthma in the following 12-month period in a primarily adult, privately insured population. Stanford, Shah, D'Souza, and Schatz (2013) found that a shorter ratio assessment period can be used to predict asthma exacerbations in the following 
12-month period, in both commercially insured and Medicaid populations.

Previous studies have used 12-month outcome periods. From a clinical standpoint and for a practical application of the ratio, demonstrating that the ratio can predict shorter-term outcomes is important. It could mean that an intervention targeted at improving ratios (through controller medication prescribing) in patients with a low ratio may lead to an immediate improvement in outcomes. Additionally, previous studies have included leukotriene receptor antagonists (LTRAs) in the ratio calculation, despite the fact that LTRAs are not preferred controller medication therapy and that LTRAs are often prescribed for allergic rhinitis in children. It is important to determine whether the inclusion of LTRAs in the calculation strengthens or weakens its predictive ability.

The objective of this study is to investigate whether the controller-to-total asthma medication ratio independently predicts hospitalizations and emergency department visits, over subsequent 3, 6, and 12 month time periods, in a Medicaid population of children with asthma. Secondary objectives include determining if the ratio is a stronger predictor with or without the inclusion of leukotriene antagonists (LTRA) as a controller, and determining what cut-point should be used in order to accurately target at-risk patients.

\section{Methods}

We conducted a retrospective analysis utilizing 2007-2009 South Carolina Medicaid data. Patients age 2-18 years with at least one calendar year of continuous Medicaid enrollment were included. All analyses were conducted using 2007 ratios predicting 2008 visits and 2008 ratios predicting 2009 visits (two year-pairs). We isolated all encounters with a primary diagnosis of asthma using International Classification of Diseases 9th Revision
(ICD-9) codes 493.00-493.02, 493.10-493.493.12, 493.20-493.22, 493.8, and 493.9. From this group, we eliminated all patients with the following co-morbid conditions using ICD-9 codes: exercise induced asthma; cystic fibrosis; bronchiolitis; Respiratory Syncytial Virus; moderate, severe, or profound mental retardation; congenital heart disease; sickle cell disease; chronic respiratory disease arising in the perinatal period; tracheostomy; gastrostomy; or any other artificial opening of the gastrointestinal tract (Gupta, Bewtra, Prosser, \& Finkelstein, 2006). From this group of children with asthma, those with persistent asthma were identified using the Healthcare Effectiveness Data and Information Set (HEDIS) criteria. This includes anyone with any of the following in one calendar year: (1) an asthma related hospitalization, (2) an asthma related ED visit, (3) 4 or more asthma medication fills, or (4) 4 or more asthma related outpatient visits and 2 or more asthma medication fills.

\section{Asthma Medication Ratio}

The controller-to-total asthma medication ratios [number of controller fills / (number of controller fills + number of short-acting beta-agonist fills)] were then calculated for each patient in years 2007 and 2008. We included any ICS or combined ICS/ Long-acting beta agonists in the ratio. Although ICSs are the preferred first line preventive therapy for children with persistent asthma, the National Heart, Lung, and Blood Institute Guidelines for the management of asthma recommend LTRAs as alternative first line therapy or adjunctive therapy for patients with persistent asthma not controlled on ICS alone (Expert Panel Report 3 [EPR-3], 2007). If patients had a pharmacy claim for more than one controller in a month they were both included in the calculation. Our ratios were calculated both with and without LTRAs included as a controller medication in order to determine if this alternative therapy contributes to the ratio's 
predictive ability. Ratios range from zero to one with one being ideal and zero being the worst (no controller). Ratios were categorized into deciles for the analyses to determine ideal cut-points.

\section{Outcome Variable}

Asthma related ED visits and hospitalizations were identified by Current Procedural Terminology (CPT) codes and ICD-9 codes. ${ }^{1}$

\section{Analysis}

Logistic regression models first determined if the ratio is a significant predictor of subsequent emergent healthcare visits and which ratio, with or without LTRA, is a stronger predictor of emergent health care visits. This was determined by comparing Areas Under the Curve (AUC) from models with the ratio as the independent variable and each of the three outcome variables (ED visit, hospitalization, and ED visit or hospitalization) as a dichotomized dependent variable. Each model was run with the ratio including LTRA and the ratio not including LTRA.

Once we determined that the ratio was a significant predictor of emergent healthcare visits and whether or not to include LTRA in the ratio, we then determined which ratio cut-point is most appropriate. We built logistic regression models with a dichotomous independent variable (ratio greater than or less than/equal to cut-point $\mathrm{X}$ where $\mathrm{X}=0.1,0.2$, etc.) and a dichotomous dependent variable of whether or not there was an emergent healthcare visit. The AUC were compared to find the cut-point that maximized the predictive ability of the ratio. These analyses were completed in both

CPT๔ codes for ED visits are 99281-99285 and for hospitalization are 99217-99223 and 99234-99236. ICD-9 codes for asthma are 493.00-493.02, 493.10-493.12, 493.20-493.22, 493.8, and 493.9. CPT only copyright 2006-2013 American Medical Association. All rights reserved. year-pairs and for all three outcome variables (ED Visit, hospitalization, ED Visit or hospitalization).

Finally, to investigate whether the controllerto-total asthma medication ratio predicts emergent health care visits over subsequent 3, 6, and 12 month time periods, multivariable logistic regression was used to determine if the ratio remained a significant predictor of ED visits, hospitalizations, and a combined outcome of ED visit or hospitalization, over each time period, controlling for age, gender, race, and rurality (defined using the Metropolitan Statistical Area definition of urban). To assess for differences in the ratio's predictive ability by race or age, we included interaction terms in the final models.

We used SAS 9.3 for all data analyses. The Medical University of South Carolina IRB approved this study.

\section{Results}

We identified 19,512 children with persistent asthma aged 2-18 years to include in the analyses. Mean age was 8.9 years (median 9.0); 58\% were male, and 55\% were black (Exhibit 1).

The controller-to-total asthma medication ratio significantly predicted subsequent ED visits alone, hospitalizations alone, and combined count of ED visits and hospitalizations in separate logistic regression analyses. While the relationship was significant both with and without LTRA, including LTRA in the ratio resulted in stronger predictive potential when compared to the ratio without LTRA. The strongest relationship was seen using the ratio with LTRA to predict hospitalizations (Exhibit 2).

Using the ratio with LTRA, and testing each decile as a potential cut-point, the ratio cut-point that maximized the AUC when predicting EDvisits, hospitalizations, and ED visit or hospitalization ranged from $0.4-0.6$ in both year-pairs. We chose a cut-point of 0.5 for the final models (Exhibit 3). 


\section{Exhibit 1. Demographics of South Carolina Medicaid Recipients with Persistent Asthma 2007-2009 ( $n=19,512)$}

\begin{tabular}{lcc}
\hline Demographic & N & (\%) \\
\hline Male & 11,401 & $(58)$ \\
Minority Race/Ethnicity $^{1}$ & 13,110 & $(67)$ \\
Urban $^{2}$ & 13,549 & $(69)$ \\
Patients with an ED visit & 4,281 & $(22)$ \\
Patients with a Hospitalization & 666 & $(3)$ \\
& Mean & Median \\
Age & 8.9 & 9.0 \\
Number of SABA ${ }^{3}$ fills & 7.4 & 5.0 \\
Number of ICS ${ }^{3}$ fills & 4.5 & 3.0 \\
Number of LTRA ${ }^{3}$ fills & 5.0 & 2.0 \\
\hline
\end{tabular}

NOTES: ${ }^{1}$ Minority race/ethnicity = self-declared black race, Hispanic ethnicity, and "other."

${ }^{2}$ Indicates individuals who live in a county that is part of a South Carolina Metropolitan Statistical Area.

${ }^{3} \mathrm{SABA}=$ Short-acting beta agonist; ICS = Inhaled Corticosteroid;

LTRA = Leukotriene Receptor Antagonist.

SOURCE: South Carolina Medicaid Data, 2007-2009.
Exhibit 3. Areas Under the Curve for Asthma Medication Ratio Cut points by Year-Pair and Outcome

\begin{tabular}{lcc}
\hline Ratio Cut-point & $\begin{array}{c}\text { Area Under } \\
\text { the Curve } \\
\text { 2007-2008 }\end{array}$ & $\begin{array}{c}\text { Area Under } \\
\text { the Curve } \\
\mathbf{2 0 0 8 - 2 0 0 9}\end{array}$ \\
\hline Hospitalization & 0.557 & 0.569 \\
0.4 & 0.590 & 0.575 \\
0.5 & 0.555 & 0.598 \\
0.6 & & \\
ED Visit & 0.561 & 0.559 \\
0.4 & 0.552 & 0.564 \\
0.5 & 0.554 & 0.566 \\
0.6 & & 0.562 \\
Hospitalization & & 0.568 \\
or ED Visit & 0.560 & 0.609 \\
0.4 & 0.559 & \\
0.5 & 0.554 & \\
0.6 & & \\
\hline NOTES: ${ }^{1}$ Ratios calculated with 2007 data predicting 2008 emergent \\
care visits. & & \\
${ }^{2}$ Ratios calculated with 2008 data predicting 2009 emergent care visits.
\end{tabular}

Exhibit 2. Logistic Regression Models ${ }^{1}$ Evaluating Asthma Medication Ratio's Ability to Predict Subsequent Emergent Healthcare Visits for Asthma

\begin{tabular}{lccc}
\hline & $\begin{array}{c}\text { Hospitalizations } \\
\text { AUC }^{2}\end{array}$ & $\begin{array}{c}\text { ED Visits } \\
\text { AUC }\end{array}$ & $\begin{array}{c}\text { Hospitalization or } \\
\text { ED Visit AUC }\end{array}$ \\
\hline 2007-2008 $^{3}$ & & & \\
Ratio with LTRA n $=13,756$ & 0.614 & 0.587 & 0.586 \\
Ratio without LTRA $\mathrm{n}=13,199$ & 0.589 & 0.554 & 0.552 \\
$\mathbf{2 0 0 8 - 2 0 0 9 4}^{4}$ & & & \\
Ratio with LTRA $\mathrm{n}=15,480$ & 0.636 & 0.589 & 0.591 \\
Ratio without LTRA $\mathrm{n}=14,711$ & 0.611 & 0.542 & 0.546 \\
\hline
\end{tabular}

NOTES: ${ }^{1}$ Models included ratio as only independent variable and $\mathrm{p}<.001$ for all models.

${ }^{2}$ Area Under the Curve.

${ }^{3}$ Ratios calculated with 2007 data predicting 2008 emergent care visits.

${ }^{4}$ Ratios calculated with 2008 data predicting 2009 emergent care visits.

SOURCE: South Carolina Medicaid Data, 2007-2009.

After controlling for age, gender, race and rurality, patients with a ratio $<0.5$ were significantly more likely to have a subsequent ED visit, hospitalization, and ED visit or hospitalization. The ratio retained its predictive ability in both year-pairs for all three outcome variables in all three time periods (3 month, 6 month, and 12 month) with the exception of the
2008 ratio not predicting 20093 month and 6 month hospitalizations. This is likely due to the rarity of this outcome. Hospitalization is a less common outcome and by decreasing the time interval to 3 and 6 months the occurrence became even more rare ( $n=48$ for 3 month hospitalizations and $\mathrm{n}=86$ for 6 month hospitalizations) (Exhibit 4). 
Exhibit 4. Logistic Regression Models ${ }^{1}$ Predicting Asthma Emergent Care Visits if Asthma Medication Ratio is $<0.5$

\begin{tabular}{ccccccc}
\hline & \multicolumn{2}{c}{ Hospitalizations } & \multicolumn{2}{c}{ ED Visits } & \multicolumn{2}{c}{ Hospitalization } \\
& Or ED Visit \\
& OR & $(95 \% \mathrm{CI})$ & OR & $(95 \% \mathrm{CI})$ & OR & $(95 \% \mathrm{CI})$ \\
\hline $\mathbf{2 0 0 7 - \mathbf { 2 0 0 8 } ^ { 2 }}$ & & & & & & \\
3 month & 1.9 & $(1.1-3.4)$ & 1.6 & $(1.2-2.0)$ & 1.6 & $(1.3-2.0)$ \\
6 month & 2.0 & $(1.3-3.0)$ & 1.5 & $(1.3-1.8)$ & 1.6 & $(1.3-1.9)$ \\
12 month & 1.8 & $(1.3-2.5)$ & 1.5 & $(1.3-1.8)$ & 1.6 & $(1.4-1.8)$ \\
$\mathbf{2 0 0 8 - 2 0 0 9}$ & & & & & & \\
3 month & 1.1 & $(.69-1.9)$ & 1.5 & $(1.2-1.9)$ & 1.5 & $(1.2-1.9)$ \\
6 month & 1.1 & $(.66-2.4)$ & 1.6 & $(1.4-2.0)$ & 1.6 & $(1.4-1.9)$ \\
12 month & 1.7 & $(1.2-2.5)$ & 1.7 & $(1.4-1.9)$ & 1.7 & $(1.5-1.9)$ \\
\hline
\end{tabular}

NOTES: ${ }^{1}$ All models controlled for age, gender, race, and rurality and all models except italicized were significant with $\mathrm{p}<.001$

${ }^{2}$ Ratios calculated with 2007 data predicting 2008 emergent care visits.

${ }^{3}$ Ratios calculated with 2008 data predicting 2009 emergent care visits.

SOURCE: South Carolina Medicaid Data, 2007-2009.

\section{Discussion}

The results of this study suggest that the controllerto-total asthma medication ratio can predict both ED visits and hospitalizations, over short as well as long term time periods, in children with persistent asthma. While the knowledge of short term predictive potential is new, these findings also confirm, in a large population of Medicaid-funded children, the findings of Stanford et al. which show that the asthma medication ratio can be used to identify children at risk for ED and hospital visits before they occur. Specifically, patients who have controllers dispensed as less than half of their asthma medications are approximately $60 \%$ more likely to have an emergent care visit in the subsequent 3,6 , and 12 month time periods. An intervention aimed at improving controller medication use in those with a ratio $<0.5$ may be an effective way to prevent future emergent healthcare visits in a period as short as three months.

We have compared the predictive power of the ratio when it is calculated both with and without the inclusion of LTRAs. We feel this is an important contribution to the literature, because of the frequency with which LTRAs are prescribed in pediatric populations, both for asthma and allergic rhinitis. One of the limitations of working with pharmacy claims data is that one cannot always link the medication claim to a visit diagnosis code. We were able to show that, regardless of prescribing intent, LTRAs strengthen the relationship between the ratio and subsequent outcomes; therefore, they should be included in the ratio calculation in future studies and when the ratio is used by providers and payers to identify high risk patients.

In South Carolina, there are over 2,490 hospitalizations (average cost \$6,192) and over 12,000 ED visits (average cost \$237) per year in children under 18 years of age (The Bureau of Community Health and Chronic Disease Prevention, 2012). The results of this study suggest that an intervention that increases controller medication use in children by increasing their ratio above 0.5 could result in 7,200 fewer ED visits and 1,495 fewer hospitalizations per year, leading to a cost savings of over $\$ 10,000,000$ per year. These cost estimates do not take into account additional savings from decreased missed work/school days 
or the improved quality of life that would be experienced by the child and family.

To our knowledge, this is the first study to demonstrate the ability of the ratio to independently predict hospitalizations and ED visits in a large population of children over 3,6 , and 12 month time periods; thereby, expanding on previous researchers' work. (Schatz et al., 2006; Stanford et al., 2013). The ability of the ratio to not only predict visits in the subsequent 12 months, but also in the subsequent 3 and 6 month time periods, is a critical basis for the practical application of the ratio. We find promising the idea that improving preventive medication use can yield short-term benefits in terms of decreased frequency of acute care visits, and we believe it provides rationale that targeted interventions that may not be sustainable over a long period of time might still have a significant impact on outcomes.

There are several limitations to this study. We analyzed Medicaid data from one state; therefore, our findings may not be generalizable to other geographic locations or to non-publicly insured patients. However, to date, this represents the largest cohort of children who have been studied with this ratio, and we feel our findings contribute significantly to the literature. It will be important in the future to test this ratio in privately insured children with asthma. Additionally, we used administrative claims data to do this study. There is inherent inaccuracy when relying on ICD-9 codes for identification of subjects. Pharmacy claims data will not capture free samples provided to patients or the intent of the medication (for example, asthma or allergic rhinitis). We feel that our sample size is large enough to minimize the effect of any potential coding inaccuracies or missed controller prescriptions. And testing this ratio in administrative claims data is critical, because the utility of this ratio and any interventions based on it will be through tracking prescription claims. The data used in this study covers through 2009. Although 2010 data was available at the time of our analysis, we do not feel that the incorporation of the extra year's data in this project would significantly affect our results. Additionally, there have not been any significant changes in the treatment of asthma during the recent 3 years. We feel that our data provides an accurate representation of the current relationship between the ratio and subsequent visits.

\section{Conclusions}

The asthma medication ratio is a significant predictor of hospitalizations and ED visits over subsequent 3,6 , and 12 month periods, in publically insured children with asthma. In addition, the ratio's predictive potential is stronger when LTRAs are included in the calculation. Providers and payers can use this ratio in real time to identify at risk patients so that they can intervene to improve controller medication use and therefore prevent emergent healthcare visits.

\section{Correspondence}

Anne Lintzenich Andrews, M.D., MSCR, Medical University of South Carolina-Pediatrics, 135 Rutledge Ave., MSC 561, Charleston, SC 29425, andrewsan@ musc.edu, T. 843-876-8512 F. 843-876-8709.

\section{Acknowledgments}

We wish to acknowledge Greg Barabell, MD Medical Director of Select Health of South Carolina for his contributions to the development of this project.

\section{Disclaimer}

License For Use Of Physicians' Current Procedural Terminology, Fourth Edition (“CPT")

CPT codes, descriptions and other data only are copyright 2013 American Medical Association. All rights reserved. CPT is a registered trademark of the American Medical Association (AMA).

You, your employees and agents are authorized to use $\mathrm{CPT}$ only as contained in the following authorized 
materials of Centers for Medicare and Medicaid Services (CMS) internally within your organization within the United States for the sole use by yourself, employees and agents. Use is limited to use in Medicare, Medicaid or other programs administered by CMS. You agree to take all necessary steps to insure that your employees and agents abide by the terms of this agreement.

Any use not authorized herein is prohibited, including by way of illustration and not by way of limitation, making copies of CPT for resale and/or license, transferring copies of CPT to any party not bound by this agreement, creating any modified or derivative work of CPT, or making any commercial use of CPT. License to use CPT for any use not authorized herein must be obtained through the AMA, CPT Intellectual Property Services, 515 N. State Street, Chicago, IL 60654. Applications are available at the AMA Web site, http://www.ama-assn.org/go/cpt.

Applicable FARS $\backslash$ DFARS Restrictions Apply to Government Use.

This product includes CPT which is commercial technical data and/or computer data bases and/or commercial computer software and/or commercial computer software documentation, as applicable which were developed exclusively at private expense by the American Medical Association, 515 North State Street, Chicago, Illinois, 60654. U.S. Government rights to use, modify, reproduce, release, perform, display, or disclose these technical data and/or computer data bases and/or computer software and/or computer software documentation are subject to the limited rights restrictions of DFARS 252.227-7015(b)(2) (November 1995) and/or subject to the restrictions of DFARS 227.7202-1(a) (June 1995) and DFARS 227.7202-3(a) (June 1995), as applicable for U.S. Department of Defense procurements and the limited rights restrictions of FAR 52.227-14 (June 1987) and/or subject to the restricted rights provisions of FAR 52.227-14 (June 1987) and FAR 52.227-19 (June 1987), as applicable, and any applicable agency FAR Supplements, for non-Department of Defense Federal procurements.

\section{AMA Disclaimer of Warranties and Liabilities.}

CPT is provided "as is" without warranty of any kind, either expressed or implied, including but not limited to, the implied warranties of merchantability and fitness for a particular purpose. No fee schedules, basic unit, relative values or related listings are included in $\mathrm{CPT}$. The AMA does not directly or indirectly practice medicine or dispense medical services. The responsibility for the content of this file/product is with CMS and no endorsement by the AMA is intended or implied. The AMA disclaims responsibility for any consequences or liability attributable to or related to any use, non-use, or interpretation of information contained or not contained in this file/product. This agreement will terminate upon notice if you violate its terms. The AMA is a third party beneficiary to this agreement.

\section{CMS Disclaimer}

The scope of this license is determined by the AMA, the copyright holder. Any questions pertaining to the license or use of the CPT should be addressed to the AMA. End Users do not act for or on behalf of the CMS. CMS DISCLAIMS RESPONSIBILITY FOR ANY LIABILITY ATTRIBUTABLE TO END USER USE OF THE CPT. CMS WILL NOT BE LIABLE FOR ANY CLAIMS ATTRIBUTABLE TO ANY ERRORS, OMISSIONS, OR OTHER INACCURACIES IN THE INFORMATION OR MATERIAL CONTAINED ON THIS PAGE. In no event shall CMS be liable for direct, indirect, special, incidental, or consequential damages arising out of the use of such information or material.

Should the foregoing terms and conditions be acceptable to you, please indicate your agreement and acceptance by clicking below on the button labeled "accept".

\section{References}

Adams, R. J., Fuhlbrigge, A., Finkelstein, J. A., Lozano, P., Livingston, J. M., Weiss, K. B., \& Weiss, S. T. (2001). Impact of inhaled antiinflammatory therapy on hospitalization and emergency department visits for children with asthma. [Clinical Trial Multicenter Study Research Support, Non-U.S. Gov't Research Support, U.S. Gov't, P.H.S.]. Pediatrics, 107(4), 706-711. PubMed http://dx.doi.org/10.1542/ peds.107.4.706 
Akinbami, L. (2006). The state of childhood asthma, United States, 1980-2005. Advance Data, (381), 1-24. PubMed

Akinbami, L. J., Moorman, J. E., Bailey, C., Zahran, H. S., King, M., Johnson, C. A., \& Liu, X. (2012). Trends in asthma prevalence, health care use, and mortality in the United States, 2001-2010. NCHS Data Brief, (94), 1-8.

Akinbami, L. J., Moorman, J. E., Garbe, P. L., \& Sondik, E. J. (2009). Status of childhood asthma in the United States, 1980-2007. Pediatrics, 123(Suppl. 3), S131-S145. PubMed http:// dx.doi.org/10.1542/peds.2008-2233C

Andrews, A. L., Teufel, R. J., II, \& Basco, W. T., Jr. (2012). Low rates of controller medication initiation and outpatient follow-up after emergency department visits for asthma. [Research Support, U.S. Gov't, P.H.S.]. The Journal of Pediatrics, 160(2), 325-330. PubMed http://dx.doi.org/10.1016/j.jpeds.2011.07.037

Cloutier, M. M., Hall, C. B., Wakefield, D. B., \& Bailit, H. (2005). Use of asthma guidelines by primary care providers to reduce hospitalizations and emergency department visits in poor, minority, urban children. [Multicenter Study Research Support, Non-U.S. Gov't]. The Journal of Pediatrics, 146(5), 591-597. PubMed http:// dx.doi.org/10.1016/j.jpeds.2004.12.017

Expert Panel Report 3 (EPR-3): Guidelines for the Diagnosis and Management of AsthmaSummary Report 2007. (2007). [Practice Guideline]. Journal of Allergy and Clinical Immunology, 120(5 Suppl), S94-138.

Finkelstein, J. A., Lozano, P., Farber, H. J., Miroshnik, I., \& Lieu, T. A. (2002). Underuse of controller medications among Medicaid-insured children with asthma. [Research Support,
Non-U.S. Gov't Research Support, U.S. Gov't, P.H.S.]. Archives of Pediatrics \& Adolescent Medicine, 156(6), 562-567. PubMed http:// dx.doi.org/10.1001/archpedi.156.6.562

Fuhlbrigge, A., Carey, V. J., Adams, R. J., Finkelstein, J. A., Lozano, P., Weiss, S. T., \& Weiss, K. B. (2004). Evaluation of asthma prescription measures and health system performance based on emergency department utilization. [Research Support, NonU.S. Gov't Research Support, U.S. Gov't, P.H.S.]. Medical Care, 42(5), 465-471. PubMed http:// dx.doi.org/10.1097/01.mlr.0000124249.84045.d7

Gupta, R. S., Bewtra, M., Prosser, L. A., \& Finkelstein, J. A. (2006). Predictors of hospital charges for children admitted with asthma. AmbulatoryPediatrics, 6(1), 15-20. http://dx.doi. org/10.1016/j.ambp.2005.07.001 PubMed

Lieu, T. A., Quesenberry, C. P., Jr., Capra, A. M., Sorel, M. E., Martin, K. E., \& Mendoza, G. R. (1997). Outpatient management practices associated with reduced risk of pediatric asthma hospitalization and emergency department visits. [Comparative Study]. Pediatrics, 100(3 Pt 1), 334-341. PubMed http://dx.doi.org/10.1542/ peds.100.3.334

Schatz, M., Zeiger, R. S., Vollmer, W. M., Mosen, D., Mendoza, G., Apter, A. J., . . Cook, E. F. (2006). The controller-to-total asthma medication ratio is associated with patient-centered as well as utilization outcomes. [Research Support, NonU.S. Gov't]. Chest, 130(1), 43-50. http://dx.doi. org $/ 10.1378 /$ chest.130.1.43

Stanford, R. H., Shah, M. B., D'Souza, A. O., \& Schatz, M. (2013). Predicting Asthma Outcomes in Commercially Insured and Medicaid Populations. The American Journal of Managed Care, 19(1), 60-67. PubMed 
The Bureau of Community Health and Chronic Disease Prevention. (2012). Asthma in South Carolina: Common, Costly and Climbing Retrieved from the South Carolina DHEC Web site at: http://www.scdhec.gov/health/epidata/ docs/stateasthma.pdf

Vernacchio, L., Trudell, E. K., \& Muto, J. M. (2013). Correlation of Care Process Measures With Childhood Asthma Exacerbations. Pediatrics.
PubMed http://dx.doi.org/10.1542/peds.20121144

Warman, K. L., Silver, E. J., \& Stein, R. E. (2001). Asthma symptoms, morbidity, and antiinflammatory use in inner-city children. [Clinical Trial Randomized Controlled Trial Research Support, Non-U.S. Gov't]. Pediatrics, 108(2), 277-282. PubMed http://dx.doi. org/10.1542/peds.108.2.277 